\title{
Competencias digitales en estudiantes de Magisterio de la primera generación del Plan Ceibal: un estudio comparado en Salto, Uruguay
}

\section{Digital skills in teaching students from the first generation of Plan Ceibal: A comparative study in Salto, Uruguay}

\author{
Lourdes Berretta y María Noel Sterla ${ }^{1}$
}

Fecha de recepción: 12 de marzo de 2020

Fecha de aceptación: 13 de diciembre de 2020

Resumen:

El presente artículo presenta los principales resultados de la investigación Desarrollo de competencias digitales en estudiantes de Magisterio en Salto. Estudio comparado entre estudiantes de Magisterio de la primera generación de Plan Ceibal y estudiantes de Magisterio que no participaron del mismo. La pregunta problema de investigación que se plantea es: ¿Cuáles son las diferencias en el desarrollo de competencias digitales entre estudiantes de Magisterio de la primera generación de implementación del Plan Ceibal y estudiantes que no participaron del mismo en Salto en el año 2017? El objetivo es comparar el desarrollo de competencias digitales entre estudiantes de Magisterio de la primera generación de implementación del Plan Ceibal y estudiantes que no participaron de este Plan. Dicha investigación se realizó en 2017 con estudiantes que cursaban segundo año de Magisterio debido a la importancia creciente de las Tecnologías Digitales en la Educación. En relación al tipo de diseño, se trató de una investigación de corte exploratorio dado que, al momento en el que se desarrolló el trabajo, no se tuvo acceso a antecedentes en relación al problema específico de investigación. Es un estudio mixto ubicado en un nivel descriptivo-comparativo con dos muestras de casos seleccionadas por conveniencia. En este artículo se abordan algunas de las veintiuna competencias analizadas en la investigación en base al Marco Común de Competencia Digital Docente de INTEF (2017). El área de competencias

\footnotetext{
${ }^{1}$ Lourdes Berretta - Maestra egresada del Instituto de Formación Docente de Salto. Docente Orientadora de Tecnologías Digitales y de Educación e Integración de Tecnologías Digitales, en el Consejo de Formación en Educación, IFD Salto. Correo electrónico: wiba888@gmail.com

María Noel Sterla - Profesora de Geografía egresada del Instituto de Formación Docente de Salto e IPA, Diploma en Geografía Udelar-CFE. Profesora en: Consejo de Educación Secundaria, Consejo de Educación Técnico Profesional y Consejo de Formación en Educación, IFD Salto. Correo electrónico: noelsterla@gmail.com
} 
analizada es información y comunicación informacional, la misma comprende competencias digitales que apuntan a identificar, localizar, obtener, almacenar, organizar y analizar información digital, evaluando su finalidad y relevancia. Entre los aportes que generó la investigación, se afirma que, en esta área analizada, existe una leve mejoría en el desarrollo de las competencias digitales en la muestra con Ceibal.

Palabras clave: Formación docente, competencias digitales, competencia informacional, Plan Ceibal.

\section{Abstract}

This article aims to disseminate some of the main results of the research: Development of digital skills in teaching students in Salto. Comparative study between Teaching students of the first generation of Plan Ceibal and Teaching students who did not participate in it. The research's problem question that arises is: What are the differences in the development of digital skills between Teacher Preparation students from the first generation of the implementation of Plan Ceibal and students who did not participate in it in Salto in 2017? The objective is to compare the development of digital competences between Teaching students from the first generation of the implementation of Plan Ceibal and students who did not participate in it. This research was carried out in 2017 with students who were in their second year of Teaching due to the growing importance of Digital Technologies in Education. Regarding the type of design this research presents, it is an exploratory type of investigation since, at the time the work was carried out, there was no access to antecedents in relation to the specific research problem. It is a mixed-study located at a descriptive-comparative level with two samples of cases selected for convenience. This article will address some of the twenty-one competencies analyzed in the research based on the Marco Común de Competencia Digital Docente of INTEF (2017). The area of competencies analyzed is information and informational communication: it includes digital competences that aim to identify, locate, obtain, store, organize and analyze digital information, evaluating its purpose and relevance. Among the contributions generated by the research, it is stated that in this analyzed area that there has been a slight improvement in the development of digital skills in the con Ceibal sample.

Keywords: Teacher training, digital skills, informational skills, Ceibal Project.

\section{Introducción}

La presente investigación estudia el impacto del Plan Ceibal en la adquisición de las competencias digitales.

Plan Ceibal, es un proyecto socioeducativo de Conectividad Educativa Básica para el Aprendizaje en Línea creado en 2007 en Uruguay con la finalidad de la inclusión e 
igualdad de oportunidades que apoye con tecnología las políticas educativas uruguayas. A partir de su implementación, cada estudiante que ingresa al sistema educativo público accede a una computadora para su uso personal con conexión a Internet gratuita desde el centro educativo. Plan Ceibal brinda un conjunto de programas, recursos educativos y capacitación docente que transforma las maneras de enseñar y aprender. (Plan Ceibal, 2007)

Al respecto, se consideró la categorización del INTEF ${ }^{2}$ (2017) que se estructura en cinco grandes áreas que, a su vez, se subdividen en veintiuna competencias. En este artículo optamos por hacer foco en el área de competencias de información y alfabetización informacional, que incluye tres competencias: navegación, búsqueda y filtrado de información, datos y contenido digital; evaluación de información, datos y contenido digital y almacenamiento; y recuperación de información, datos y contenido digital.

Dicha investigación surgió en el contexto del trabajo docente en los espacios de intercambio, en un ámbito en el que se observaron diversas posturas y perspectivas, debido a la inquietud por mejorar las prácticas estudiantiles y docentes en relación al uso de las tecnologías en la educación.

El trabajo fue desarrollado por un equipo multidisciplinario de docentes del Instituto de Formación Docente de Salto Rosa Silvestri, perteneciente al Consejo de Formación en Educación en Uruguay. Este equipo estuvo integrado por: Cecilia Arbiza, Lourdes Berretta, Angelina Domínguez, Leonardo Martinelli, Ana Elena Olivera, Alexis Reyes y Noel Sterla.

\section{Planteamiento del problema}

La pregunta de investigación se planteó de la siguiente manera: ¿Cuáles son las semejanzas y diferencias en el desarrollo de competencias digitales entre estudiantes de Magisterio de la primera generación de implementación del Plan Ceibal y estudiantes que no participaron del mismo en Salto, Uruguay, en el año 2017 ?

\section{Objetivos de la investigación:}

Comparar el desarrollo de competencias digitales entre estudiantes de Magisterio de la primera generación de implementación del Plan Ceibal y estudiantes que no participaron del Plan en Salto, año 2017.

Para dar cumplimiento al objetivo general, se plantearon los siguientes objetivos específicos:

${ }^{2}$ Instituto Nacional de Tecnologías Educativas y de Formación del Profesorado. 
- Identificar las competencias digitales que desarrollan los estudiantes de Magisterio de la primera generación de implementación del Plan Ceibal, en Salto, en el año 2017.

- Reconocer las competencias digitales que desarrollan los estudiantes de Magisterio que no participaron del Plan Ceibal, en Salto, en el año 2017.

- Establecer semejanzas y diferencias en el desarrollo de competencias digitales observado en cada uno de los grupos de estudiantes mencionados.

Antecedentes:

Para comenzar el trabajo de investigación se consideraron varias fuentes internacionales y nacionales.

En relación a las fuentes internacionales y vinculado al desarrollo de las competencias digitales, Gallego, Gámiz y Gutiérrez (2010) sostienen que la percepción de los estudiantes sobre su dominio y adquisición de estas es menor frente a las competencias didáctico-pedagógicas que necesitan para su práctica docente, ellos son conscientes de la necesidad de la formación para su futuro desempeño profesional.

En cuanto a las fuentes nacionales, se realizó un estudio exploratorio con docentes de primaria indagando si la implementación del Plan Ceibal cambiaba las prácticas de los docentes en Salto (Fernández, et al., 2008). Además, se analizó el impacto del Plan Ceibal en el aprendizaje de la matemática y la lectura (De Melo, Machado, Miranda, y Viera, 2013).

Durante los meses de octubre y noviembre del año 2008, se llevó a cabo en el departamento de Salto un estudio exploratorio por parte de docentes y estudiantes del Centro Regional de Profesores del Litoral, el mismo abarcó a 210 docentes de primaria, quienes respondieron a una encuesta. Su objetivo fue explorar si la implementación del Plan Ceibal cambiaba las prácticas de los docentes. En este estudio concluyeron: «No es posible afirmar que los docentes están cambiando sus prácticas como consecuencia del programa de innovación» (Fernández, et al., 2008). Esta investigación se realizó en el mismo año en que se entregaron las primeras xo en el departamento de Salto, cuando aún el impacto del Plan estaba dado, más por la llegada de las xo, que por el uso que se podría realizar de ellas. La xo es una computadora portátil que pretende facilitarles a los estudiantes el acceso al conocimiento y oportunidades de explorar, experimentar y expresarse. El proyecto una computadora por niño, OLPC (del inglés One Laptop Per Child), ha sido desarrollado con software libre y abierto, su sistema operativo es Sugar. En Uruguay, se la nombra como ceibalita, en referencia al Plan Ceibal que la distribuyó a todos los escolares y docentes del sistema educativo público (Da Silva, y López, 2014). 
En el año 2011, se analiza el impacto del Plan Ceibal en el aprendizaje de la matemática y la lectura, y los estudiantes de Salto ingresan en esa muestra. De los resultados se desprende que la introducción de tecnología en el aula tuvo un impacto positivo en el desempeño de los niños en matemática.

En este estudio, en relación a la motivación hacia la escuela, la gran mayoría de los niños estaban contentos; consideraban que en su clase tenían buenos amigos y les daría pena tener que cambiar de centro educativo. Si bien en todos los casos mencionados las respuestas fueron levemente superiores para los niños con introducción de tecnología en el aula mediante Plan Ceibal, no hubo indicios respecto a que el Plan genere un estado de motivación particular respecto al entorno escolar. No obstante, sus padres y maestras opinaron mayoritariamente que los niños se encontraban más motivados para ir a la escuela y hacia el trabajo diario, desde que tenían su XO (Ferrando, Machado, Perazzo y Bernengo, 2011).

En la investigación de De Melo., Machado, Miranda y Viera (2013) se indaga sobre los efectos del Plan Ceibal, centrándose en los resultados académicos alcanzados por un panel de estudiantes en las áreas de matemática y lectura, así como sus efectos en los hábitos de estudio y en la percepción individual sobre diversas habilidades cognitivas. De esta surge que, si bien hay resultados positivos en matemática, los mismos no son significativos (se ubica en el entorno de 0.2 desvíos respecto a la media). Por su parte, en lengua, no se observan resultados académicos positivos. Además, surgen algunos otros indicadores como que el uso de Internet para la realización de tareas domiciliarias no aparece como relevante, pues en ese momento Internet no era accesible en la mayoría de los hogares y el uso de las xo en escuelas de contexto crítico era mayor que en las demás. Esto se atribuye a un colectivo docente más joven, lo que puede indicar que sean más propensos a la innovación en el aula.

En relación a las competencias digitales en la formación inicial docente, en la Universidad de Granada, España, Gallego, Gámiz y Gutiérrez (2010) realizan una investigación tomando una muestra de estudiantes de la Titulación de Maestros en la Facultad de Ciencias de la Educación, cuyas edades se encuentran en el rango de veintiún y veinticuatro años. Dicha investigación revela, entre otros aspectos, que la percepción de los estudiantes sobre su dominio y adquisición en competencias digitales es menor frente a las competencias didáctico-pedagógicas que necesitan para su práctica docente y que los mismos son conscientes de su necesidad de formación en estos aspectos para su futuro desempeño profesional.

En la misma temática centrada en las competencias digitales y la actitud entre estudiantes de la Universidad de Extremadura, España, Centeno y Cubo (2013) afirman que el uso de las TIC se ha generalizado de tal modo que el $100 \%$ de los estudiantes disponen de recursos vinculados con ellas. Se destaca el importante 
papel que posee el ámbito académico en la adquisición y desarrollo de las competencias digitales.

Del recorrido realizado en investigaciones en nuestro país, es posible identificar algunas en lo que se refiere al impacto de las xo en el aprendizaje de lengua y matemática, pero no se han estudiado en relación a las competencias digitales previo a nuestra investigación. Hoy se tiene ya una generación que ha transitado por la educación obligatoria siendo a la vez beneficiarios del Plan Ceibal. Con anterioridad a nuestro trabajo, solamente una de las investigaciones relevadas se centraba en el tema específico competencias digitales, siendo esta en España, país en el cual, hasta la fecha del artículo, no se había implementado un plan similar al Plan Ceibal.

\section{Fundamentación teórica:}

Para el abordaje de este artículo, se profundiza en la formación inicial docente y su vinculación con las competencias digitales. El cometido de este estudio fue explorar si los estudiantes de formación docente magisterial, quienes fueron partícipes de una política educativa innovadora (Plan Ceibal) durante su formación primaria y secundaria, desarrollaron las competencias digitales mencionadas en relación a los que no participaron.

En lo relativo a la formación de los docentes, se consideró a Bosco (2008), quien presenta reflexiones sobre la formación del profesorado en tecnologías digitales en términos de las competencias a desarrollar y la perspectiva metodológica aconsejable a esa formación, si lo que se pretende es promover esa capacidad docente. Este autor sostiene que la sociedad actual es cultural y tecnológicamente compleja, de modo que nos enfrenta a replantearnos la formación inicial en educación como un elemento clave y estratégico para enfrentar retos y problemas que antes no existían. Algunos de los retos son el cambio continuo, la proliferación de información, la generación de conocimiento nuevo y el uso de tecnologías digitales. También plantea que se hace imprescindible definir una serie de nuevas competencias digitales para el ejercicio de la docencia, puesto que ellas implican el uso consciente de artilugios electrónicos. Sostiene que no es suficiente la consulta de material multimedia, sino que es necesaria su integración con sentido en una secuencia didáctica específica.

Bosco (2008) trabaja algunos conceptos y para ello se remite a la UNESCO (2008) afirmando que:

los docentes en ejercicio necesitan estar preparados para proporcionar a sus estudiantes oportunidades de aprendizaje apoyadas en las TIC, ayudándoles a sacar provecho de estas herramientas a favor de un aprendizaje reflexivo, basado en la resolución de problemas y en la generación de conocimiento. Para ello 
reivindican el uso de recursos como las simulaciones interactivas, los recursos educativos digitales abiertos y los instrumentos sofisticados de recolección y análisis de datos. Todas opciones consideradas como las más indicadas para el desarrollo de la comprensión y el pensamiento crítico. Es decir, no solo no se queda en un enfoque instrumental en lo que a competencias en Tic a desarrollar se refiere, sino que avanza en un modo particular de uso y desarrollo de propuestas pedagógicas basadas en TIC, enfatizando aquellas que permiten el desarrollo del aprendizaje autónomo. (Bosco, 2008, pp. 4-5)

El docente que no ha recibido esta formación inicial necesita capacitarse en los aspectos indicados, superar la adquisición instrumental y llegar a la apropiación simbólica, poniendo a las TIC al servicio de mejores aprendizajes. El profesor que está capacitado evalúa materiales, recursos y propuestas didácticas; reformula contenidos y estrategias; reflexiona sobre su práctica y la modifica en un proceso de revisión continua.

En relación a la práctica docente, Litwin (en Azinian, 2009) sostiene que las nuevas tecnologías no solo han proporcionado a los docentes nuevas posibilidades para desarrollar sus estrategias de enseñanza, sino que han aportado a los estudiantes ayuda para reconocer sus propios procesos de comprensión. También afirma que, cuando los docentes enfrentan el desafío de trabajar con las tecnologías y reflexionar sobre la enseñanza, encuentran que este proceso es una importante experiencia formativa, una oportunidad para su propio desarrollo profesional. Litwin llama residuo pedagógico al nuevo conocimiento emergente cuando los docentes construyen experiencias educativas a partir de la reflexión sobre las implicancias pedagógicas del uso de las tecnologías y de las características de las estrategias de enseñanza.

En otro orden, se tiene en consideración que solo un docente formado en la reflexión puede tomar decisiones apropiadas para el rediseño de los entornos de aprendizaje que requiere la integración curricular de las TIC, con el fin de producir una transformación cualitativa de las prácticas pedagógicas. Como fue expresado anteriormente, la formación inicial docente se vincula con las competencias digitales en nuestro estudio al explorar si los estudiantes de Magisterio las han desarrollado durante su formación anterior.

Pérez Gómez (2012) sostiene que la educación en competencias surge frente a la necesidad de la nueva sociedad que demanda una educación flexible, interdisciplinaria y/ que sustituya a la educación enciclopédica que se ha visto interceptada con el ingreso de las Tecnologías de Información y Comunicación en el aula. 
El concepto de competencia es, para Tardif (2003), un saber actuar complejo que se apoya sobre la movilización y la utilización eficaces de una variedad de recursos. En este sentido, una competencia está lejos de un objetivo y ella no es sinónimo de saber-hacer o de un conocimiento procedural. La idea de saber actuar hace surgir la noción de que cada competencia está esencialmente ligada a la acción y le otorga un carácter global. Es importante la integración en la definición de la movilización y la utilización eficaz de un conjunto de recursos. Una competencia no constituye una forma de algoritmo memorizado y practicado repetidamente en vista a asegurar la perennidad y la reproducción, sino un saber actuar muy flexible y adaptable a diversos contextos y problemáticas. Una competencia es más bien de orden heurístico que algorítmico. La flexibilidad y la adaptabilidad de la competencia justifican plenamente su movilización.

Ferrari (2012), citado por INTEF (2017), sostiene que la competencia digital es un conjunto de conocimientos, habilidades y actitudes necesarias para ser funcional en el actual entorno digital. Plantea que la adquisición de competencias digitales le permite al usuario adaptarse a las necesidades de las tecnologías e interaccionar socialmente y le brinda la posibilidad de utilizarla para una mejor práctica profesional.

Según INTEF (2017), otra conceptualización es «el uso crítico y seguro de las Tecnologías de la Sociedad de la Información para el trabajo, el tiempo libre y la comunicación» (p. 12). En ella se consideran las siguientes áreas de competencias digitales: área 1: Información y alfabetización informacional, área 2: Comunicación y colaboración, área 3: Creación de contenidos digitales, área 4: Seguridad, área 5: Resolución de problemas.

En este artículo se destaca los resultados de la investigación en el área 1: información y alfabetización informacional que pretende identificar, localizar, obtener, almacenar, organizar y analizar información digital, evaluando su finalidad y relevancia. Las competencias vinculadas a esta área son: navegación, búsqueda y filtrado de información, datos y contenido digital; evaluación de información, datos y contenido digital; almacenamiento y recuperación de información, datos y contenido digital.

Asociado al área de información y alfabetización informacional, existe el fenómeno de la hipertextualidad. Esto es planteado por Area Moreira cuando expresa:

Por otra parte, la presentación de la información a través de las nuevas tecnologías se caracteriza por su ruptura con el modelo de organización lineal de la cultura impresa; la imagen, el sonido, el hipertexto revelan una forma distinta de publicarla. Frente a las formas tradicionales de acceso a la información que son secuenciales (p.e. la visualización de una película, o la lectura de un libro) las 
llamadas tecnologías digitales almacenan la información de modo tal que no existe una única secuencia de acceso a la misma, sino que las distintas unidades o segmentos de información están entrelazados a través de nodos similares a una red. $(2009$, p. 38)

\section{Diseño metodológico}

Esta investigación se desarrolló a partir de un diseño de tipo exploratorio dado que, al momento en que se desarrolló el trabajo, no se accedió a antecedentes en relación al problema específico de investigación. Esto supone que este estudio exploratorio puede generar conocimientos que originen nuevos recorridos para futuras investigaciones y que, al profundizar en torno a las dimensiones del objeto de estudio, amplíen la comprensión del mismo. Por otra parte, el estudio también tuvo carácter evaluativo, ya que, al comparar estudiantes que participaron del Plan Ceibal desde su comienzo y estudiantes que no participaron, se consideró el efecto del mismo en el desarrollo de las competencias digitales.

Para el diseño, se incorporaron técnicas de recolección de corte cuantitativo y cualitativo. Por un lado, se aplicaron instrumentos de corte cuantitativo y evaluativo, considerando las respuestas a través formularios que se fueron cuantificando. En relación al enfoque cualitativo, con técnicas de observación e interpretación, se pretendió hacer un estudio con una mirada microsocial que profundizara en la problemática y no en la generalización de resultados. Esto es, en la generación de conocimientos en torno a procesos específicos referidos a nuestra muestra.

Las técnicas de recolección de datos utilizadas fueron la observación participante y los instrumentos de evaluación de competencias. Además, se utilizó un cuestionario definido que consta de un formulario y actividades prácticas, lo que permitió la triangulación de datos como criterio de validación; posibilitando, así, una elaboración descriptiva del fenómeno y una aproximación al conocimiento de su incidencia en el desarrollo de las competencias digitales.

El diseño de los instrumentos estuvo orientado de acuerdo a las categorías y dimensiones que componen la temática abordada. En ese sentido, se presenta la siguiente tabla que da cuenta del proceso de operacionalización, partiendo de las áreas de competencias digitales a estudiar y el desglose de las mismas en diferentes niveles, de lo más abstracto a lo más concreto.

\section{Tabla 1}

Desarrollo del Proceso de Construcción de los Instrumentos, Pasaje de Competencias en Variables y de Variables en Indicadores.

\begin{tabular}{|c|l|l|l|l|}
\hline Conceptos & Dimensión & Variable & Indicadores & Instrumentos \\
\hline
\end{tabular}


Berretta y Sterla - Competencias digitales en estudiantes de Magisterio de la primera...

\begin{tabular}{|c|c|c|c|c|}
\hline \multirow{4}{*}{$\begin{array}{l}\text { Información y } \\
\text { alfabetización } \\
\text { informacional }\end{array}$} & \multirow{2}{*}{\begin{tabular}{l}
\multicolumn{1}{c}{ Navegación, } \\
búsqueda y filtrado de \\
información datos y \\
contenido digital
\end{tabular}} & $\begin{array}{l}\text { Uso de } \\
\text { navegadores web. }\end{array}$ & \begin{tabular}{l}
\multicolumn{1}{c}{ Identifica los } \\
navegadores. \\
$\quad$ Los utiliza \\
correctamente \\
reconociendo \\
barra de \\
direcciones y barra \\
de herramientas.
\end{tabular} & $\begin{array}{l}\text { Evaluación. } \\
\text { Actividad } \\
\text { práctica de aula. }\end{array}$ \\
\hline & & \begin{tabular}{l}
\multicolumn{1}{c}{ Saber } \\
seleccionar la \\
información \\
necesaria.
\end{tabular} & \begin{tabular}{l}
\multicolumn{1}{c}{ Identifica } \\
diferentes \\
buscadores. \\
Formula \\
palabras clave o \\
frases concretas \\
para la búsqueda.
\end{tabular} & $\begin{array}{l}\text { Evaluación. } \\
\text { Bloque uso de } \\
\text { Internet. }\end{array}$ \\
\hline & $\begin{array}{l}\text { Evaluación de } \\
\text { información, datos y } \\
\text { contenido digital. }\end{array}$ & $\begin{array}{l}\text { Es relevante en } \\
\text { su situación de } \\
\text { búsqueda de } \\
\text { información. }\end{array}$ & $\begin{array}{l}\quad \text { Reúne la } \\
\text { información } \\
\text { seleccionada, la } \\
\text { procesa, } \\
\text { comprende y } \\
\text { evalúa en forma } \\
\text { crítica. (planilla) }\end{array}$ & $\begin{array}{c}\text { Actividad } \\
\text { práctica de aula. }\end{array}$ \\
\hline & $\begin{array}{l}\text { Almacenamiento y } \\
\text { recuperación de } \\
\text { información, datos y } \\
\text { contenido digital. }\end{array}$ & $\begin{array}{l}\text { Guarda } \\
\text { información digital. } \\
\text { Organiza la } \\
\text { información } \\
\text { Localiza la } \\
\text { información } \\
\text { guardada } \\
\text { (almacenada). }\end{array}$ & $\begin{array}{l}\text { Clasifica la } \\
\text { información. } \\
\text { Crea carpetas. } \\
\text { Nombra los } \\
\text { archivos en } \\
\text { relación al tema. } \\
\text { Recupera la } \\
\text { información. }\end{array}$ & $\begin{array}{c}\text { Actividad } \\
\text { práctica de aula. }\end{array}$ \\
\hline
\end{tabular}

Fuente: Recuperado de la investigación Desarrollo de competencias digitales en estudiantes de Magisterio en Salto. Estudio comparado entre estudiantes de Magisterio de la primera generación de Plan Ceibal y estudiantes de Magisterio que no participaron del mismo.

Se trabajó con dos unidades de observación que componen la población: los estudiantes de magisterio del IFD Salto en el año 2017, discriminándose así en muestra Con Ceibal y Sin Ceibal.

Muestra 1: estudiantes de segundo año que hayan participado del Plan Ceibal durante su formación escolar y liceal teniendo la posibilidad de desarrollar, con sus docentes, actividades curriculares que incluyeran las tecnologías digitales en su práctica favoreciendo, de esta manera, el desarrollo de competencias digitales en Salto en el año 2017.

Muestra 2: estudiantes de segundo año que no hayan participado del Plan Ceibal durante su formación escolar y liceal recibiendo computadoras ni participando en 
actividades curriculares con docentes que incluyeran las tecnologías digitales en su práctica procurando el desarrollo de las competencias digitales en Salto, año 2017.

Para la validación de los resultados de la investigación, se recurrió a diferentes estrategias de triangulación (Stake, 2005) de forma de contrastar las interpretaciones para fortalecer su rigurosidad, pero también para dar lugar a otras interpretaciones posibles. Se trabajó con triangulación metodológica cuantitativa-cualitativa con distintos instrumentos de recolección de datos como la observación, el cuestionario autoadministrado y la evaluación de actividades prácticas. Otra de las estrategias que contribuyó con la validación de datos estuvo dada por la triangulación de investigadores, se trabajó con más de un investigador en las distintas instancias del trabajo de campo y en la aplicación de los diferentes instrumentos de recogida de datos.

\section{Resultados}

Entre los aportes que generó la investigación, se afirma que, en el área analizada, Información y alfabetización informacional, existe una leve mejoría en el desarrollo de las competencias digitales en la muestra con Ceibal. A continuación, se presenta una tabla de datos que se utilizó para comparar ambas muestras en las competencias analizadas:

Tabla 2

Competencias involucradas con el área de información

\begin{tabular}{|c|c|c|c|}
\hline Área & Competencia & Muestra con CEIBAL & Muestra sin CEIBAL \\
\hline & $\begin{array}{l}\text { Navegación, } \\
\text { búsqueda y filtrado } \\
\text { de información, } \\
\text { datos y contenido } \\
\text { digital. }\end{array}$ & $\begin{array}{l}\text { Mayor conocimiento en } \\
\text { la diferenciación entre } \\
\text { buscador y navegador, pero } \\
\text { no diferencian } r \text { una } \\
\text { dirección web de una } \\
\begin{array}{l}\text { dirección de } \\
\text { electrónico. }\end{array}\end{array}$ & $\begin{array}{l}\text { Mayor confusión entre } \\
\text { navegador y buscador, } \\
\text { independientemente de ello, } \\
\text { reconocen y diferencian una } \\
\text { dirección web de un correo } \\
\text { electrónico. }\end{array}$ \\
\hline Información & $\begin{array}{l}\text { Evaluación de } \\
\text { información, datos } \\
\text { y contenido digital. }\end{array}$ & $\begin{array}{l}\text { Demuestra mayor } \\
\text { sistematización en el uso } \\
\text { específico de buscadores } \\
\text { para conseguir resultados } \\
\text { adecuados a sus intereses, } \\
\text { un estudiante utiliza } \\
\text { específicamente Google } \\
\text { académico obteniendo } \\
\text { resultados más asertivos. }\end{array}$ & $\begin{array}{l}\text { Demuestra menor } \\
\text { sistematización en el uso } \\
\text { específico de buscadores } \\
\text { para conseguir resultados } \\
\text { adecuados a sus intereses. } \\
\text { Utiliza palabras claves sin } \\
\text { diferenciar el objetivo } \\
\text { específico de la búsqueda. }\end{array}$ \\
\hline
\end{tabular}


Berretta y Sterla - Competencias digitales en estudiantes de Magisterio de la primera...

\begin{tabular}{|l|l|lr|l|}
\hline & $\begin{array}{l}\text { Almacenamient } \\
\text { o y recuperación } \\
\text { de información, } \\
\text { datos y contenido } \\
\text { digital. }\end{array}$ & $\begin{array}{l}\text { Se } \\
\text { conocimiento de la } \\
\text { especificidad del software y y las herramientas } \\
\text { de las } \\
\text { virtuales. }\end{array}$ & $\begin{array}{l}\text { Poco conocimiento de la } \\
\text { especificidad del software y } \\
\text { desconocimiento total en } \\
\text { herramientas virtuales. }\end{array}$ \\
\hline
\end{tabular}

Fuente: Recuperado de la investigación Desarrollo de competencias digitales en estudiantes de Magisterio en Salto. Estudio comparado entre estudiantes de Magisterio de la primera generación de Plan Ceibal y estudiantes de Magisterio que no participaron del mismo.

Realizada la comparación entre las muestras con Ceibal y sin Ceibal, se desglosa lo evaluado en el área de competencias. En primer lugar, en relación a la competencia navegación, búsqueda y filtrado de información, datos y contenido digital, se observan diferencias entre las muestras. La muestra con Ceibal sabe diferenciar entre navegador web y buscador web. La muestra sin Ceibal reconoce y diferencia entre una dirección web y un correo electrónico luego de acceder a los links, necesita navegarlos para comprender la diferencia entre ambos.

En cuanto a la competencia evaluación de información, datos y contenido digital, la muestra con Ceibal indica que hay mayor sistematización en el uso específico de buscadores para conseguir resultados adecuados a sus intereses; sin embargo, la muestra sin Ceibal presenta menor desarrollo en ese sentido, en tanto que utiliza palabras claves que no diferencian el objetivo específico de la búsqueda. Las palabras claves contribuyen con una búsqueda en la web más específica, contemplando la importancia del desarrollo de competencias digitales en el marco de la web semántica.

En el análisis de la competencia almacenamiento y recuperación de información, datos y contenido digital, la muestra con Ceibal evidencia conocimiento y especificidad de software (ofimática) además de herramientas virtuales; sin embargo, la muestra sin Ceibal demuestra poco conocimiento.

Si bien los estudiantes de la muestra con Ceibal tienen mayor desarrollo en líneas generales, no podemos ser concluyentes en cuanto a que el Plan Ceibal las haya ampliado.

\section{Las competencias más desarrolladas en estudiantes destinatarios del Plan Ceibal}

En relación a la navegación, búsqueda y filtrado de información, datos y contenido digital, de las respuestas al instrumento utilizado (formulario de evaluación), surge que la muestra con Ceibal diferencia entre buscador y navegador web; en la muestra, solo uno de los estudiantes no diferencia entre una y otra, la mayoría cita 
de forma correcta. Los estudiantes respondieron de forma adecuada la diferencia entre URL y correo electrónico sin necesidad de navegar al destino.

En relación a la utilización de buscadores, esta muestra utiliza palabras específicas para realizar las búsquedas, por lo que llega a resultados más precisos. Están más habituados a buscar libremente en la red, demuestran mayor solvencia al utilizar palabras precisas para acortar las búsquedas tal como lo expresa Area Moreira (2009) cuando refiere a las búsquedas en el contexto de la hipertextualidad.

En relación a la competencia almacenamiento y recuperación de información, datos y contenido digital, los estudiantes de esta muestra crean carpetas temáticas y guardan los archivos (video, imagen o texto) en el escritorio.

\section{Dificultades que presentan los estudiantes de la muestra sin Plan Ceibal}

En esta muestra los estudiantes no diferencian entre URL y correo electrónico antes de acceder al sitio, necesitan navegar los dos enlaces sugeridos para responder.

En cuanto a la utilización de buscadores, las palabras utilizadas no evidencian especificidad. Por ejemplo, al buscar el concepto desarrollo para Psicología en la realización de la actividad práctica, no tienen en cuenta que el buscador incluirá desarrollo en todos los aspectos posibles si no incorporan otras palabras claves como psicología y autor. Además, han utilizado más libros en formato papel, buscan en índices, se puede interpretar que presentan menos recorridos propios de la hipertextualidad. Esto incide en el tipo de búsquedas que realizan.

En relación a la competencia almacenamiento y recuperación de información, datos y contenido digital, guardan los archivos en el escritorio sin crear carpetas. Ante la pregunta de los observadores de por qué guardan en el escritorio, responden: «Por qué así lo encuentro enseguida», «Sé dónde está» (Observación P).

Se podría decir que, en relación a la gestión y almacenamiento de la información en la computadora, presentan más dificultades para resolverlo. Al observar las computadoras pertenecientes a los evaluados de dicha muestra, se percibe que todo lo que producen lo guardan en el escritorio. Uno de los estudiantes afirma: «a la pc la uso a los sopapos, el celular lo uso mejor y es más práctico; en la pc no sé dónde guardo las cosas» (Observación, L).

\section{Las competencias que no mostraron diferencias entre ambos grupos}

En ambas muestras los estudiantes diferencian adecuadamente entre página web y correo electrónico, aunque los estudiantes de la muestra sin Ceibal necesitaron cliquear en ambas URL para establecer la diferencia. 
Además, utilizan exclusivamente el buscador Google. Durante la observación de las actividades realizadas surge la interrogante a los encuestados de por qué utilizan exclusivamente ese buscador. Algunas respuestas ante esa interrogante son: «¿Cuál otro si abre en Google?» (Observación, L) y «A veces uso Google Académico» (Observación, M). Es el buscador que se abre por defecto en la mayoría de los equipos al utilizar como navegador Chrome.

Teniendo en cuenta las respuestas al formulario de la encuesta y a las actividades prácticas realizadas, el equipo de investigación creó una categoría utilizando tres niveles: básico $0 \%$ a $33 \%$, intermedio de $33 \%$ a $66 \%$ y avanzado de $66 \%$ a $100 \%$.

Previo a la tabulación de datos y su estudio, se evaluó el grado de desarrollo de competencias observándose que la muestra sin Ceibal respondió mejor debido a la frecuencia del uso del buscador Google, mientras que la muestra con Ceibal utilizó más el navegador.

A modo de síntesis, en relación a las competencias del área analizada, nos referiremos a los niveles alcanzados de estudiantes relevados (28) entre las dos muestras. En el área de información y alfabetización informacional, competencia navegación, búsqueda y filtrado de información y contenido digital: la muestra con Ceibal se encuentra en el nivel avanzado (diez de doce estudiantes evaluados contestaron correcto, un 83,3\%), mientras que la muestra sin Ceibal (once de dieciséis estudiantes contestaron adecuadamente, un $68,7 \%$ ) se halla en el nivel intermedio.

Si se considera la competencia evaluación de información, datos y contenido digital, ambas muestras se ubican en el nivel intermedio (de la muestra con Ceibal, siete de doce estudiantes contestaron de forma correcta, alcanzando un $58.3 \%$ y seis de dieciséis de la muestra sin Ceibal lo hicieron de forma acertada, un $37.5 \%$ ).

Sin embargo, en la competencia almacenamiento y recuperación de información, datos y contenido digital, la muestra con Ceibal se sitúa en el nivel avanzado (83\%), mientras que la sin Ceibal se sitúa en nivel intermedio (56\%).

El siguiente cuadro grafica el desarrollo de esta área de competencia digital para las dos muestras.

Tabla 3

Nivel de desarrollo de competencias digitales en el área de competencias de Información y alfabetización informacional.

\begin{tabular}{|l|l|l|}
\hline & Muestra & Muestra SC \\
\hline
\end{tabular}


Berretta y Sterla - Competencias digitales en estudiantes de Magisterio de la primera...

\begin{tabular}{|c|c|c|}
\hline $\begin{array}{c}\text { Navegación, búsqueda y filtrado de información y contenido } \\
\text { digital. }\end{array}$ & Avanzado & Intermedio \\
\hline Evaluación de información, datos y contenido digital & Intermedio & Intermedio \\
\hline $\begin{array}{c}\text { Almacenamiento y recuperación de información, datos y } \\
\text { contenido digital }\end{array}$ & Avanzado & Intermedio \\
\hline
\end{tabular}

Fuente: Recuperado de la investigación Desarrollo de competencias digitales en estudiantes de Magisterio en Salto. Estudio comparado entre estudiantes de Magisterio de la primera generación de Plan Ceibal y estudiantes de Magisterio que no participaron del mismo.

\section{Discusión de resultados}

En reiteradas oportunidades del trabajo de investigación, se identificaron situaciones en las que inciden más de una competencia digital. Si bien las áreas de competencias están clasificadas teóricamente por separado, el análisis de los datos da cuenta de cómo se integran en la práctica, no se comportan de forma independiente. Esta cuestión se percibe con mayor claridad en la muestra con Ceibal.

El objeto de estudio de la investigación resultó ser un fenómeno complejo, con múltiples variables a considerar, que va más allá de la influencia de dicho Plan como la masificación de los teléfonos smartphone, el boom de las redes sociales, el acceso masivo a Internet en los hogares, entre otras.

Ambas muestras refieren a la facilidad que ofrece el celular, ya que no requiere de «descargar, guardar, buscar la información», únicamente un «re enviar» es suficiente para llegar al objetivo. Prefieren soluciones rápidas, lo inmediato, aquello que no requiere una inversión de tiempo más que el necesario «El celular es más fácil y cómodo de usar que una computadora» (muestra CC, Observación, P.).

\section{Conclusiones}

En cuanto a la navegación, búsqueda y filtrado de información, datos y contenido digital, surge de los datos recogidos que los estudiantes de la muestra con Ceibal presentan mayor desarrollo que los de la muestra sin Ceibal.

Los estudiantes de la muestra con Ceibal presentan un nivel superior al momento de reconocer y diferenciar entre buscador y navegador web, dirección web y dirección de correo electrónico, sin necesidad de acceder previamente a cada uno de ellos, mientras que los de la muestra sin Ceibal ingresan en cada caso para verificar si realizan el procedimiento correspondiente. 
Los estudiantes de ambas muestras trabajan exclusivamente con el buscador Google, aparentemente porque era el disponible en los equipos. Los estudiantes de la muestra con Ceibal dan cuenta de mayor desarrollo para realizar búsquedas, dado que utilizan palabras específicas y llegan a resultados más precisos, mientras que los de la muestra sin Ceibal utilizan palabras que no evidencian especificidad.

Otro elemento que marca esta diferencia en el desarrollo refiere a cómo gestionan el almacenamiento de información presentando mayor nivel organizacional los estudiantes de la muestra con Ceibal, pues nombran las carpetas que crean.

El hecho de que el Plan Ceibal haya sido implementado contemplando franjas de edades y niveles educativos implicó que la muestra sin Ceibal permaneciera fuera de la modalidad "uno a uno», no siendo incluida en la escuela ni en el liceo; aunque contará con la asignatura Informática en la etapa liceal. Para esta muestra, la tecnología no fue partícipe de su trayectoria por la educación formal, esta no se integró en general en otras aulas específicas, ni en las asignaturas de modo transversal.

En suma, esta investigación evidenció que la muestra con Ceibal ha alcanzado levemente niveles superiores en el desarrollo de competencias del área de información y alfabetización informacional. No obstante, es necesario considerar que además del uso de los dispositivos del Plan Ceibal hay otras variables que influyen en el desarrollo de estas competencias. En este sentido, se trató que las muestras fueran próximas: la última generación sin Ceibal y la primera generación con Ceibal, para que se acorte la influencia que impacte directamente en ambas muestras.

\section{Bibliografía}

Area Moreira, M. (2009). Manual electrónico: Introducción a la tecnología educativa. San Cristóbal de La Laguna, España: Universidad de La Laguna.

Arbiza, C., Berretta, L., Domínguez, A., Martinelli, L., Olivera, A. Reyes y A., Sterla, M. (2017). Desarrollo de competencias digitales en estudiantes de Magisterio en Salto. Estudio comparado entre estudiantes de Magisterio de la primera generación de Plan Ceibal y estudiantes de Magisterio que no participaron del mismo. Salto, Uruguay: inédito.

Azinian, H (2009) "Las tecnologías de la información y la comunicación en las prácticas pedagógicas». "Manual para organizar proyectos". Buenos Aires, Argentina: Ediciones Novedades Educativas.

Bosco, A. (2008). Las tecnologías de la información y la comunicación en la formación del profesorado: lineamientos, actualidad y prospectiva. Razón y Palabra. Recuperado de: https://www.redalyc.org/pdf/1995/199520798002.pdf

Centeno, G. y Cubo, S. (2013). Evaluación de la competencia digital y las actitudes hacia las TICs del alumnado universitario. Revista de Investigación Educativa, 31(2), 517-536. 
Da Silva, M., López, L. (2014). Pensando el «PLAN CEIBAL» desde la perspectiva de la acción pública y la teoría del actor-red. Montevideo, Uruguay: UDELAR

De Melo, G., Machado, A, Miranda, A y Viera, M. (2013). Profundizando en los efectos del Plan Ceibal. Instituto de Economía-FCEyA-UdelaR / Centro de Investigación y Docencia Económicas (CIDE) México. A.N.E.P.

Fernández, F; et al. (2008). Estudio exploratorio sobre la percepción del impacto del Plan Ceibal ¿Cambian las prácticas de los docentes?. Salto, Uruguay: departamento de Sociología de la Educación, área Ciencias de la Educación del Centro Regional de Profesores del Litoral.

https://es.scribd.com/document/210816637/PlanCeibalArt-C3-ADculo2908-Salto.

Ferrando, M. , Machado, A., Perazzo, I. y Bernengo, A. (2011). Aprendiendo con las xo: El impacto del Plan Ceibal en el aprendizaje». Montevideo, Uruguay: Instituto de Economía Universidad de la República, Serie Documentos de Trabajo DT $3 / 11$.

Gallego, M., Gámiz, V., y Gutiérrez, E. (2010). El futuro docente ante las competencias en el uso de las tecnologías de la información y la comunicación para enseñar. Revista Electrónica Educativa. Número 34. Recuperado de: https://www.researchgate.net/publication/258210172_El_futuro_docente_ante_las competencias_en_el_uso_de_las_tecnologias_de_la_informacion_y_la_comunic acion para ensenar

Instituto Nacional de Tecnologías Educativas y de Formación del Profesorado (INTEF). (2017). Marco Común de Competencia Digital Docente. Recuperado el 23 de junio de 2017, de https://aprende.intef.es/sites/default/files/2018-05/2017_1020_Marco-Com\%C3\% BAn-de-Competencia-Digital-Docente.pdf.

Pérez Gómez, A. (2012). Educarse en la era digital. Madrid, España: Morata, S. L.

Plan Ceibal (2007). Qué es el Plan Ceibal. Recuperado en 2020/11/14 de https://www.ceibal.edu.uy/es/institucional

Stake, R.E (2005). «Investigación con estudios de casos». Madrid, España: Morata Tardif, J. (2003). Desarrollo de un programa por competencias: De la intención a la puesta en marcha. Traducido de Pedagogie collégiale, 16(3) Mars (por Óscar Corvalán). 